International Journal of Pure and Applied Mathematics

Volume 99 No. 3 2015, 227-244

ISSN: 1311-8080 (printed version); ISSN: 1314-3395 (on-line version)

url: http://www.ijpam.eu

doi: http://dx.doi.org/10.12732/ijpam.v99i3.1

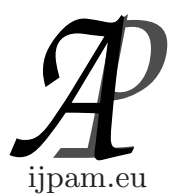

\title{
AMEANBLE ACTION AND INVARIANT MEAN
}

\author{
Kankeyanathan Kannan \\ Department of Mathematics and Statistics \\ University of Jaffna, \\ Jaffna, SRI LANKA
}

\begin{abstract}
We have been thinking also about the possibility of getting a direct proof of Invariant Approximation Property for amenable groups that uses some averaging procedure and does not use von Neumann algebras as in John Roe book. This sounds that like there is a special version of the Invariant Approximation Property (IAP) for von Neumann algebras which is different to the standard one.
\end{abstract}

AMS Subject Classification: 20F65, 18G60

Key Words: amenable, uniform Roe algebras, invariant approximation property

\section{Introduction}

We assume that the reader is familiar with the basic notions in operator algebras and operator spaces, see Roe [9], Anantharaman-Delaroche [1], and Kannan [5], [6], [7] and [8] for the details on the invariant approximation property. We say that the uniform Roe algebra, $C_{u}^{*}(G)$, is the $C^{*}$ - algebra completion of the algebra of bounded operators on $\ell^{2}(X)$ which have finite propagation.

According to Roe [9] $G$ has the invariant approximation property (IAP) if

$$
C_{r}^{*}(G)=C_{u}^{*}(G)^{G}
$$

In this paper, we will check that for amenable groups that use some averaging

Received: July 16, 2014

(c) 2015 Academic Publications, Ltd. url: www.acadpubl.eu 
procedure, and that do not use Von Neumann algebras not passing Invariant Approximation Property. We can define invariant elements in $C_{u}^{*}(G)$ using the fact that $G$ is amenable. We want to use an averaging procedure as in BNW (see [3] and [2]). The main purpose of this paper is to prove that the Theorem:

Theorem 1.1. The amenable group does not have IAP for von Neumann algebras which is different to the standard one.

\section{Preliminaries}

Coarse geometry is the study of the large scale properties of spaces. The notion of large scale is quantified by means of a coarse structure. First we recall the following definitions:

Definition 2.1. (see [9]) Let $X, Y$ be metric spaces and $f: X \longrightarrow Y$ a not necessarily continuous map.

1. The map $f$ is called coarsely proper (or metrically proper), if the inverse image of a bounded set is bounded.

2. The map $f$ is called coarsely uniform (or uniformly bornologous), if for every $r>0$ there is $s(r)>0$ such that for all $x_{1}, x_{2}$ in $X$

$$
d\left(x_{1}, x_{2}\right) \leq r \Longrightarrow d\left(f\left(x_{1}\right), f\left(x_{2}\right)\right) \leq s(r) .
$$

3. The map $f$ is called a coarse map, if it is coarsely proper and coarsely uniform.

4. Let $S$ be a set. Two maps $f, g: S \longrightarrow X$ are called close if there is $C>0$ such that for all $s$ in $S$

$$
d(f(s), g(s))<C .
$$

5. A subset $E$ of $X \times X$ is called controlled (or entourage), if the coordinate projection maps $\pi_{1}, \pi_{2}: E \longrightarrow X$ are close.

Definition 2.2. (see [9]) A coarse structure on a set $X$ is a collection of subsets of $X \times X$, called the controlled sets or entourages for the coarse structure, which contains the diagonal and is closed under the formation of subsets, inverses, products, and (finite) unions.

It is easy to see that the controlled sets associated to a metric space $X$ have the following properties: 
1. Any subset of a controlled set is controlled;

2. The transpose $E^{t}=\{(x, y):(y, x) \in E\}$ of a controlled set $\mathrm{E}$ is controlled;

3. The composition $E_{1} \circ E_{2}$ of controlled sets $E_{1}$ and $E_{2}$ is controlled; where $E_{1} \circ E_{2}:=(x, z) \in X \times X: \exists y \in X,(x, y) \in E_{1}$ and $(y, z) \in E_{2}$;

4. A finite union of controlled sets is controlled;

5. The diagonal $\Delta_{X}:=\{(x, x): x \in X\}$ is controlled.

Definition 2.3. (see [9]) Let $(X, d)$ be a metric space, we say the metric $d$ induces a coarse structure on $X$, which is called a bounded coarse structure. More precisely, we can define the bounded coarse structure induced by the metric $d$ as follows: Set

$$
D_{r}:=\{(x, y) \in X \times X: d(x, y)<r\} .
$$

Then $E \subseteq X \times X$ is controlled, if $E \subseteq D_{r}$ for some $r>0$.

The following is an example of coarse structure.

Example 2.4. (see [9]) Let $G$ be a finitely generated group. Then the bounded coarse structure associated to any word metric on $G$ is generated by the diagonals

$$
\Delta_{g}=\{(h, h g): h \in G\}
$$

as $g$ runs over $G$.

Let us briefly recall basic definitions and facts concerning positive and negative type kernels and functions.

Definition 2.5. Let $\mathrm{X}$ be a set. A symmetric kernel on $X$ is a function $f: X \times X \longrightarrow \mathbb{R}$ with $f(x, y)=f(y, x)$

Definition 2.6. (see [9]) A kernel $f$ has conditionally positive type if for all $m \in \mathbb{N}$, all $m$-tuples $x_{1}, x_{2}, \ldots, x_{m}$ of points of $X$ and for all real scalars $\lambda_{1}, \lambda_{2}, \ldots, \lambda_{m}$, one has

$$
\sum_{i, j=1}^{m} \lambda_{i} \lambda_{j} f\left(x_{i}, x_{j}\right) \geq 0 .
$$

Definition 2.7. (see [9]) A kernel $f$ has conditionally negative type if for all $m \in \mathbb{N}$, all $m$-tuples $x_{1}, x_{2}, \ldots, x_{m}$ of points of $X$, and for all real scalars $\lambda_{1}, \lambda_{2}, \ldots, \lambda_{m}$ such that $\sum \lambda_{i}=0$, one has

$$
\sum_{i, j} \lambda_{i} \lambda_{j} f\left(x_{i}, x_{j}\right) \leq 0 .
$$


A conditionally negative kernel on a group $G$ is a conditionally negative kernel on the set of elements of $G$ such that for any $g, h, k$, in $G$,

$$
f(g h, g k)=f(h, k) .
$$

The following result in [9], which relates positive and negative type kernels, is known as Schoenberg's Lemma.

Lemma 2.8. (see [9]) Let $f$ be a symmetric kernel on a space $X$. The following statements are equivalent.

1. The kernel $f$ is of negative type.

2. For each $t>0$ the kernel $\exp (-t f)$ is of positive type.

Remark 2.9. (see [9]) Let $G$ be a group; by definition the positive function on $G$ defined by $\phi: G \longrightarrow \mathbb{R},(x, y) \longmapsto \phi\left(x^{-1} y\right)$, is a kernel of positive type

We next recall some basic fact about uniform Roe algebra and metric property of a discrete group. Next we recall the following definitions; Let $X$ be a discrete metric space.

Definition 2.10. (see [9]) We say that discrete metric space $X$ has bounded geometry if for all $R$ there exists $N$ in $\mathbb{N}$ such that for all $x \in X,\left|B_{R}(x)\right|<N$, where $B(x, r)=\{x \in X: d(y, x) \leq r\}$.

Definition 2.11. (see [9]) A kernel $\phi: X \times X \longrightarrow \mathbb{C}$

- is bounded if there, exists $M>0$ such that $|\phi(s, t)|<M$ for all $s, t \in X$

- has finite propagation if there exists $R>0$ such that $\phi(s, t)=0$ if $d(s, t)>R$.

Let $B(X)$ be a set of bounded finite propagation kernels on $X \times X$. Each such $\phi$ defines a bounded operator on $\ell^{2}(X)$ via the usual formula for matrix multiplication

$$
\phi * \zeta(s)=\sum_{r \in G} \phi(s, r) \zeta(r) \text { for } \zeta \in \ell^{2}(X) .
$$

Next, we show the operator associated with a bounded kernel is bounded.

Lemma 2.12. (see [8]) Let $X$ be bounded geometry metric space. An operator associated with a bounded finite propagation kernel is bounded.

We shall denote the finite propagation kernels on $X$ by $A^{\infty}(X)$.

Definition 2.13. The uniform Roe algebra of a metric space $X$ is the closure of $A^{\infty}(X)$ in the algebra $B\left(\ell^{2}(X)\right)$ of bounded operators on $X$. 
If a discrete group $G$ is equipped with its bounded coarse structure introduced in Example 2.4 then one can associated with it uniform Roe algebra $C_{u}^{*}(G)$ by repeating the above. Next we recall the left and right regular representation: An important class of $C^{*}$ - algebras arise in the study of groups. Let $G$ be a discrete group, then the characteristic function $\delta_{g}(s)$ of $g, s \in G$ is defined as follows [4]:

$$
\delta_{g}(s)= \begin{cases}1 & \text { if } g=s \\ 0 & \text { if } g \neq s\end{cases}
$$

If we assume that the $G$ is a discrete group then the functions $\delta_{g}$ form a basis for the Hilbert space $\ell^{2}(G)$ of square summable functions on $G$.

The group ring $\mathbb{C}[G]$ consists of all finitely supported complex-valued functions on $G$, that is of all finite combinations

$$
f=\sum_{s \in G} a_{s} s
$$

with complex coefficients.

The convolution product and the adjoint are defined as follows:

$$
\begin{gathered}
\left(\sum_{s \in G} a_{s} s\right)\left(\sum_{t \in G} a_{t} t\right)=\sum_{s, t \in G} a_{s} a_{t} s t \\
\left(\sum_{s \in G} a_{s} s\right)^{*}=\sum_{s \in G} \overline{a_{s}} s^{-1} .
\end{gathered}
$$

Denote by $B\left(\ell^{2}(G)\right)$ the $C^{*}$ - algebra of all bounded linear operator on the Hilbert space $\ell^{2}(G)$. We may distinguish between the left regular representation, which is induced by the left multiplication action, and the right regular representation, which is comes from the multiplication on the right.

Definition 2.14. (see [4]) The left regular representation

$$
\lambda: \mathbb{C}[G] \rightarrow B\left(\ell^{2}(G)\right)
$$

is defined by

$$
\lambda(s) \delta_{t}(r)=\delta_{t}\left(s^{-1} r\right)=\delta_{s t}(r) \text { for } s, r \in G .
$$

The right regular representation is given by

$$
\rho(s) \delta_{t}(r)=\delta_{t}(r s)=\delta_{t s^{-1}}(r) \text { for } s, r \in G .
$$


The left regular representation is implemented using the familiar convolution formula

$$
\left(\delta_{g} *_{\lambda} \delta_{h}\right)(s)=\sum_{t \in G} \delta_{g}\left(s t^{-1}\right) \cdot \delta_{h}(t)=\delta_{g h}(s) .
$$

It follows that for any function $f \in \ell^{2}(G)$ the left action by $\delta_{g}$ is given by

$$
\left(\delta_{g} *_{\lambda} f\right)(s)=\sum_{t \in G} \delta_{g}\left(s t^{-1}\right) . f(t)=f\left(g^{-1} s\right) .
$$

We can define the following right convolution:

$$
\left(\delta_{g} *_{\rho} \delta_{h}\right)(s)=\sum_{t \in G} \delta_{g}\left(t^{-1} s\right) \cdot \delta_{h}(t)=\delta_{h g}(s),
$$

which gives rise to the right regular representation:

$$
\left(\delta_{g} *_{\rho} f\right)(s)=\sum_{t \in G} \delta_{g}\left(t^{-1} s\right) \cdot f(t)=f\left(s g^{-1}\right) .
$$

We note that:

$$
\begin{aligned}
\delta_{g} *_{\rho} \delta_{h}(s) & =\sum_{t \in G} \delta_{g}\left(t^{-1} s\right) \cdot \delta_{h}(t) \\
& =\sum_{t \in G} \delta_{h}\left(s\left(\left(t^{1}\right)^{-1}\right) \cdot \delta_{g}\left(t^{1}\right)\right. \\
& =\delta_{h} *_{\lambda} \delta_{g}(s),
\end{aligned}
$$

and hence:

$$
\delta_{g} *_{\rho} \delta_{h}(s)=\delta_{h} *_{\lambda} \delta_{g}(s) .
$$

Proposition 2.15. The left and right representations commute, that is for all $s, t \in G$ :

$$
\rho(s) \lambda(t)=\lambda(t) \rho(s) .
$$

Proof. We have:

$$
\begin{aligned}
\rho(s) \lambda(t) \delta_{r} & =\rho(s) \delta_{t r} \\
& =\delta_{t r s^{-1}} \\
& =\lambda(t) \delta_{r s^{-1}} \\
& =\lambda(t) \rho(s) \delta_{r} .
\end{aligned}
$$

Thus

$$
\rho(s) \lambda(t)=\lambda(t) \rho(s) .
$$


Remark 2.16. The left regular representation $\lambda$ of the group ring $\mathbb{C}[G]$ assigns to each element $f \in \mathbb{C}[G]$ a bounded operator $\lambda(f)$ which acts on any $\zeta \in \ell^{2}(G)$ by convolution:

$$
\lambda(f)(\zeta)=f * \zeta .
$$

and

$$
\lambda\left(f^{*}\right)=(\lambda(f))^{*} .
$$

The image $\lambda(\mathbb{C}[G])$ of the group ring under the left regular representation is a $*$ - subalgebra of the algebra $B\left(\ell^{2}(G)\right)$ of bounded operators on $\ell^{2}(G)$.

Lemma 2.17. The left and right regular representations $\lambda$ and $\rho$ are $*-$ homomorphisms.

Proof. Let $f, g \in \mathbb{C}[G]$,

$$
\lambda(f)(\zeta)=f * \zeta \text { and } \lambda(g)(\zeta)=g * \zeta .
$$

Consider

$$
\begin{aligned}
\lambda(f * g)(\zeta) & =(f * g) * \zeta \\
& =f *(g * \zeta) \\
& =f *(\lambda(g) \zeta) \\
& =(\lambda(f) \lambda(g)(\zeta)
\end{aligned}
$$

Thus

$$
\lambda(f * g)=\lambda(f) \lambda(g) \text { for all } f, g \in \mathbb{C}[G] .
$$

Thus $\lambda$ satisfies the product. Consider

$$
\begin{aligned}
(\lambda(f)+\lambda(g)(\zeta) & =\lambda(f)(\zeta)+\lambda(g)(\zeta) \\
& =f * \zeta+g * \zeta \\
& =(f+g) * \zeta \\
& =\lambda(f+g)(\zeta)
\end{aligned}
$$

Thus

$$
\lambda(f+g)=\lambda(f)+\lambda(g) \text { for all } f, g \in \mathbb{C}[G] .
$$

Thus $\lambda$ satisfies the sum. It is easy to prove scalar multiplication, and adjoint. Therefore $\lambda$ satisfies the properties of an $*-$ homomorphisms. The proof for $\rho$ is similar. 
Lemma 2.18. The left and right regular representations $\lambda$ and $\rho$ are unitary bounded representations.

Proof. Let us define an operator

$$
\lambda_{g}: \ell^{2}(G) \longrightarrow \ell^{2}(G)
$$

which for any function $\zeta \in \ell^{2}(G)$ is given by

$$
\lambda_{g} \zeta(t)=\left(\delta_{g} * \zeta\right)(t)=\zeta\left(g^{-1} t\right)
$$

We have

$$
\begin{aligned}
\left\langle\lambda_{g} \zeta, \eta\right\rangle & =\sum_{t \in G} \lambda_{g} \zeta(t) \overline{\eta(t)} \\
& =\sum_{t \in G} \zeta\left(g^{-1} t \overline{\eta(t)}\right. \\
& =\sum_{t^{\prime} \in G} \zeta\left(t^{\prime}\right) \overline{\eta\left(g t^{\prime}\right)} \\
& =\left\langle\zeta, \lambda_{g^{-1}} \eta\right\rangle .
\end{aligned}
$$

This means that

$$
\lambda_{g}^{*}=\lambda_{g^{-1}}
$$

We have for every $g \in G, \quad \zeta \in \ell^{2}(G)$ :

$$
\begin{aligned}
\left\|\lambda_{g} \zeta\right\|^{2} & =\sum_{t \in G}\left|\zeta\left(g^{-1} t\right)\right|^{2} \\
& =\sum_{t \in G}|\zeta(t)|^{2} \\
& =\|\zeta\|^{2}
\end{aligned}
$$

therefore, $\lambda_{g}$ is a unitary bounded representation. The proof for $\rho$ is similar.

Lemma 2.19. The left regular representation $\lambda$ is a faithful representation.

Proof. Let us assume that, for some $f \in \mathbb{C}[G]$,

$$
\lambda\left(f^{*} * f\right)\left(\delta_{g}\right)(s)=0, \quad \forall g, s \in G .
$$


Then using the fact that $\lambda$ is a $*-$ homomorphisms we have that

$$
\begin{aligned}
\lambda\left(f^{*} * f\right)\left(\delta_{g}\right)(s) & =\lambda\left(f^{*}\right) \cdot \lambda(f) \delta_{g}(s) \\
& =\sum_{t \in G} f^{*}(t)\left(\lambda(f) \delta_{g}\right)\left(t^{-1} s\right) \\
& =\sum_{t \in G} \sum_{t^{\prime} \in G} f^{*}(t) f\left(t^{\prime} \delta_{g}\right)\left(t^{\prime}\right)^{-1}\left(t^{-1} s\right) \\
& =\sum_{t \in G} \sum_{t^{\prime} \in G} f\left(t^{-1}\right) f\left(t^{-1} s g^{-1}\right) \\
& =\|f\|_{2}^{2}
\end{aligned}
$$

From this we deduce that $\|f\|_{2}=0$, and so $f=0$, which implies that $\lambda$ is faithful.

The same argument can be used to show that $\rho$ is a faithful representation as well.

The reduced $C^{*}$ - algebra $C_{\lambda}^{*}(G)$ of a group $G$ (which we shall assume to be discrete) arises from the study of the left regular representation $\lambda$ of the group ring $\mathbb{C}[G]$ on the Hilbert space of square-summable functions on the group.

Definition 2.20. (see [4]) The reduced group $C^{*}-$ algebra $G$, denoted by $C_{\lambda}^{*}(G)$ is the completion of $\mathbb{C}[G]$ in the norm given, for $c \in \mathbb{C}[G]$, by

$$
\|c\|_{\lambda}=\|\lambda(c)\|
$$

The reduced $C^{*}$ - algebra $C_{\rho}^{*}(G)$ of a group $G$ (which we shall assume to be discrete) arises from the study of the right regular representation $\rho$ of the group ring $\mathbb{C}[G]$ on the Hilbert space of square-summable functions on the group.

Definition 2.21. The reduced group $C^{*}-$ algebra $G$, denoted by $C_{\rho}^{*}(G)$ is the completion of $\mathbb{C}[G]$ in the norm given, for $c \in \mathbb{C}[G]$, by

$$
\|c\|_{\rho}=\|\rho(c)\| .
$$

A discrete group $G$ has a natural coarse structure which allows us to define the uniform Roe algebra $C_{u}^{*}(G)$. A group $G$ can be equipped with either the left or right-invariant of the metric. A choice of one of the determines whether $C_{\lambda}^{*}(G)$ or $C_{\rho}^{*}(G)$ is a sublagebra of the uniform Roe algebra $C_{u}^{*}(G)$ of $G$ as we now explain. First we show that if the metric on $G$ is right-invariant then

$$
C_{\lambda}^{*}(G) \subset C_{u}^{*}(G)
$$


Let $d_{1}$ be the right-invariant metric on $G$

$$
d_{1}(x, y)=d_{1}(x g, y g) \forall g \in G \text {. }
$$

For every $g \in G$, the operator $\lambda(g)$ is given by the matrix.

Let:

$$
A_{g}^{\lambda}(x, y)= \begin{cases}1, & \text { if } x=y g \\ 0, & \text { otherwise. }\end{cases}
$$

Indeed,

$$
\begin{aligned}
A_{g}^{\lambda} \delta_{t}(s) & =\sum_{y \in G} A_{g}^{\lambda}(s, y) \delta_{t}(y) \\
& =\delta_{t}\left(g^{-1} s\right) \\
& =\delta_{g t}(s) .
\end{aligned}
$$

Note that $A_{g}^{\lambda}$ is right-invariant

$$
A_{g}^{\lambda}(x t, y t)= \begin{cases}1, & \text { if } x t=y g t \Longleftrightarrow x=y g, \\ 0, & \text { otherwise. }\end{cases}
$$

Therefore:

$$
A_{g}^{\lambda}(x, y)=A_{g}^{\lambda}(x t, y t)
$$

If the metric on $G$ is right-invariant, $A_{g}^{\lambda}$ is of finite propagation and $A_{g}^{\lambda} \in C_{u}^{*}(G)$, because $A_{g}^{\lambda}(x, y)$ is non-zero when $y^{-1} x=g$ and so

$$
d_{1}(x, y)=d_{1}\left(y^{-1} x, e\right)=d_{1}(g, e)
$$

Hence any element of $\mathbb{C}[G]$ will give use to finite propagation and this assignment extends to an inclusion

$$
C_{\lambda}^{*}(G) \hookrightarrow C_{u}^{*}(G)
$$

Next we show that if the metric on $G$ is left-invariant then

$$
C_{\rho}^{*}(G) \subset C_{u}^{*}(G)
$$

Let $d_{1}$ be the left-invariant metric on $G$

$$
d_{1}(x, y)=d_{1}(g x, g y) \forall g \in G \text {. }
$$


For every $g \in G$, the operator $\rho(g)$ is given by the matrix.

Let:

$$
A_{g}^{\rho}(x, y)= \begin{cases}1, & \text { if } x=g y \\ 0, & \text { otherwise. }\end{cases}
$$

Indeed,

$$
\begin{aligned}
A_{g}^{\rho} \delta_{t}(s) & =\sum_{y \in G} A_{g}^{\rho}(s, y) \delta_{t}(y) \\
& =\delta_{t}\left(s g^{-1}\right) \\
& =\delta_{t g}(s) .
\end{aligned}
$$

Note that $A_{g}^{\rho}$ is left-invariant

$$
A_{g}^{\rho}(t x, t y)= \begin{cases}1, & \text { if } t x=t g y \Longleftrightarrow x=g y, \\ 0, & \text { otherwise }\end{cases}
$$

Therefore:

$$
A_{g}^{\rho}(x, y)=A_{g}^{\rho}(t x, t y)
$$

If the metric on $G$ is right-invariant, $A_{g}^{\rho}$ is of finite propagation and $A_{g}^{\rho} \in C_{u}^{*}(G)$, because $A_{g}^{\rho}(x, y)$ is non-zero when $x y^{-1}=g$ and so

$$
d_{1}(x, y)=d_{1}\left(x y^{-1}, e\right)=d_{1}(g, e) .
$$

Hence any element of $\mathbb{C}[G]$ will give use to finite propagation and this assignment extends to an inclusion

$$
C_{\rho}^{*}(G) \hookrightarrow C_{u}^{*}(G)
$$

Let us now choose a right invariant metric for $G$ so that $C_{\lambda}^{*}(G) \hookrightarrow C_{u}^{*}(G)$. The right regular representation $\rho$ gives use to the adjoint action on $C_{u}^{*}(G)$ defined by

$$
A d \rho(g) T=\rho(g) T \rho(g)^{*}=\rho(g) T \rho(g)^{-1}
$$

for all $t \in G, T \in C_{u}^{*}(G)$. Our remarks above show that elements of $C_{\lambda}^{*}(G)$ are invariant with respect to this action and so $C_{\lambda}^{*}(G)$ is contained in invariant subalgebra $C_{u}^{*}(G)^{G}$.

Lemma 2.22. If $T \in C_{u}^{*}(G)$ has kernel $A(x, y)$, then $A d \rho(t) T$ has kernel $A(x t, y t)$ 
Proof. We have that:

$$
\begin{aligned}
(A d \rho(t) T \zeta)(s) & =\rho(t)\left(T \rho(t)^{*} \zeta\right)(s) \\
& =T \rho(t)^{*} \zeta(s t) \\
& =\sum_{x \in G} A(s t, x)\left(\rho(t)^{-1} \zeta\right)(x) \\
& =\sum_{x \in G} A(s t, x) \zeta\left(x t^{-1}\right)
\end{aligned}
$$

Now $A(s t, x)$ is non-zero whenever $x, y, t \in G$ are such that $y=x t^{-1}$, so $x=y t$ and we have

$$
(A d \rho(t) T \zeta)(s)=\sum_{x \in G} A(s t, y t) \zeta(y)
$$

Thus, $A d \rho(t) T$ has kernel $A(s t, y t)$.

In general, if $T \in C_{u}^{*}(X)$ then $\forall x, y \in G$ :

$$
\begin{aligned}
\left\langle A d(\rho(t)) T \delta_{x}, \delta_{y}\right\rangle & =\left\langle\rho(t) T \rho\left(t^{-1}\right) \delta_{x}, \delta_{y}\right\rangle \\
& =\left\langle T \rho\left(t^{-1}\right) \delta_{x}, \rho\left(t^{-1}\right) \delta_{y}\right\rangle \\
& =\left\langle T \delta_{x t}, \delta_{y t}\right\rangle .
\end{aligned}
$$

So the operator $T$ is $A d \rho$ - invariant if and only if

$$
\forall x, y \in X \forall t \in G\left\langle T \delta_{x t}, \delta_{y t}\right\rangle=\left\langle T \delta_{x}, \delta_{y}\right\rangle .
$$

We now define the invariant approximation: property (IAP)

Definition 2.23. (see [9]) We say that $G$ has the invariant approximation property $(I A P)$ if

$$
C_{\lambda}^{*}(G)=C_{u}^{*}(G)^{G}
$$

\section{Amenable Action and Invariant Mean}

For example, if a finite group $G$ acts on a vector space $V$, then $\forall v \in V$

$$
\bar{V}=\sum_{g \in G} g v
$$

is invariant under action on $G$.

For a discrete group $G$, we write $\operatorname{Prob}(G)$ for the space of all probability measures on $\ell^{1}(G)$. 
Definition 3.1. We define

$$
\operatorname{Prob}(G)=\left\{\mu \in \ell^{1}(G): \mu(g) \in[0,1] \text { s.t } \sum_{g \in G} \mu(g)=1\right\} .
$$

The group $G$ acts on $\operatorname{Prob}(G)$ by the left translation:

$$
(s \mu)(t)=\mu\left(s^{-1} t\right) \text { for } s, t \in G \text { and } \mu \in \operatorname{Prob}(G) .
$$

For a countable discrete group $G$ this is equivalent to the Reiter condition [3] and $[2]$.

Definition 3.2. We say that $G$ is amenable if and only if for each $g \in G$ and each $n \in \mathbb{N}$ there is an element $f_{n}(g) \in \operatorname{Prob}(G)$ of finite support with

1. $h f_{n}(g)=f_{n}(h g)$,

2. for all $g_{0}, g_{1},\left\|f_{n}\left(g_{1}\right)-f_{n}\left(g_{0}\right)\right\|_{\ell^{1}} \longrightarrow 0$ as $n \longrightarrow \infty$.

We shall denote by $\ell^{1}(G)^{*}$ the space of probability measures on $G$ and by $\ell^{2}(G)$ the unit sphere of $\ell^{2}(G)$. For $f: G \longrightarrow \mathbb{C}$ and $t \in G$ the function $t f$ is defined by $(t f)(s)=f\left(t^{-1} s\right)$.

Definition 3.3. (see [3] and [2]) We say that an invariant mean on a countable discrete group $\mathrm{G}$ is a positive linear functional on $\ell^{\infty}(G)$ which is normalised by the requirement that it pairs with the constant function 1 to give 1, and which is fixed by the natural action of $G$ on the space $\ell^{\infty}(G)^{*}$. This means that we define linear functional $\mu: \ell^{\infty}(G) \longrightarrow \mathbb{C}$ on $\ell^{\infty}(G)$, which satisfies the following conditions:

1. $\mu\left(x^{*} x\right) \geq 0 \forall x \in \ell^{\infty}(G)$,

2. $\|\mu\|=1 \mu\left(x^{*} x\right) \geq 0 \forall x \in \ell^{\infty}(G)$,

3. $\mu(g v)=\mu(v) \forall x \in \ell^{\infty}(G) \forall g \in G$.

We define the invariant kernels on $G \times G$ :

$$
f \longmapsto u_{f}(x, y)=f\left(x^{-1} y\right),
$$

where $f \in \mathbb{C}[G]$, which is left invariant, and

$$
\begin{aligned}
u_{f}(h x, h y) & =f\left(x^{-1} h^{-1} h y\right) \\
& =f\left(x^{-1} y\right)
\end{aligned}
$$




$$
=u_{f}(x, y)
$$

if $u_{f}(h x, h y)=u_{f}(x, y)$. Then

$$
\begin{aligned}
\left(A d \rho(t) u_{f}\right)(x, y) & =u_{f}(h x, h y) \\
& =u_{f}(x, y) .
\end{aligned}
$$

We have been thinking also about the possibility of getting a direct proof of Invariant Approximation Property for amenable groups that uses some averaging procedure and does not use von Neumann algebras as in John Roe book [9]. This sounds that like there is a special version of the Invariant Approximation Property(IAP) for von Neumann algebras which is different to the standard one. The main puropose of this paper is to prove that the Theorem.

Theorem 3.4. The amenable group have IAP for von Neumann algebras which is different to the standard one.

Proof. First, we need to show that

$$
C_{\lambda}^{*}(G) \subseteq C_{u}^{*}(G)^{G}
$$

if

$$
T \in C_{u}^{*}(G)^{G}=\overline{R(G)}^{G}
$$

then

$$
T \notin C_{\lambda}^{*}(G)
$$

Let

$$
\bar{u}_{n}(x, y) \in C_{\lambda}^{*}(G) .
$$

Thus $C_{\lambda}^{*}(G)$ is given by left invariant kernels. Right invariant kernels are $A d \rho(t)-$ invariant.

The family of $\left\{f_{n}\right\}$ of functions as an approximation of $\mu$ : Since $\ell^{1}(G)^{*} \cong$ $\ell^{\infty}(G)$ and also

$$
\ell^{\infty}(G)^{*} \cong \ell^{1}(G)^{* *} \supseteq \ell^{1}(G)
$$

$\mu$ is the weak $-*-$ topology. We think of $\mu$ as being given by the limit of $\left\{f_{n}\right\}$. We define

$$
\bar{u}_{n}(x, y)=\sum_{g \in G} f_{n}\left(x y^{-1}\right)(g) u(x g, y g) .
$$

We show that these kernels converge to an invariant kernel.

$$
A d \rho(t) \bar{u}_{n}(x, y)=\bar{u}_{n}(x t, y t)
$$




$$
\begin{aligned}
& =\sum_{g \in G} f_{n}(x t)(y t)^{-1}(g) u(x t g, y t g) \\
& =\sum_{g \in G} f_{n}\left(x y^{-1}\right)(g) u(x t g, y t g) \\
& =\sum_{g \in G} f_{n}\left(x y^{-1}\right)\left(t^{-1} g\right) u(x g, y g) \\
& =\sum_{g \in G}\left(t f_{n}\left(x y^{-1}\right)\right)(g) u(x g, y g) \\
& =\sum_{g \in G} f_{n}\left(t x y^{-1}\right)(g) u(x g, y g),
\end{aligned}
$$

So

$$
\sum_{g \in G}\left|f_{n}\left(t x y^{-1}\right)(g)-f_{n}\left(x y^{-1}\right)(g)\right||u(x g, y g)|<M\left\|f_{n}\left(t x y^{-1}\right)-f_{n}\left(x y^{-1}\right)\right\|_{\ell^{1}}
$$

Thus

$$
\left|A d \rho(t) \bar{u}_{n}(x, y)-\bar{u}_{n}(x, y)\right|<M\left\|f_{n}\left(t x y^{-1}\right)-f_{n}\left(x y^{-1}\right)\right\|_{\ell^{1}}
$$

By Lemma 2.12,

$$
\exists M>0 \text { s.t }|u(x g, y g)|<M \text { for all } g \in G
$$

Since $G$ is amenable, by Reiter condition (see Definition 3.2)[3] and [2]

$$
\text { for all } g_{0}, g_{1},\left\|f_{n}\left(g_{1}\right)-f_{n}\left(g_{0}\right)\right\|_{\ell^{1}} \longrightarrow 0 \text { as } n \longrightarrow \infty
$$

This means:

$$
A d \rho(t) \bar{u}_{n}(x, y)=\bar{u}_{n}(x, y)
$$

Therefore

$$
\bar{u}_{n}(x, y) \in C_{u}^{*}(G)^{G} .
$$

This implies

$$
C_{\lambda}^{*}(G) \subseteq C_{u}^{*}(G)^{G}
$$

if

$$
T \in C_{u}^{*}(G)^{G}=\overline{R(G)}^{G}
$$

where $R(G)$ is the Roe kernel and $T$ is invariant. Then

$$
\forall g \in G A d\left(\rho_{g}\right)(T)=\rho_{g} T \rho_{g}{ }^{-1}
$$


where $T \in C_{u}^{*}(G)$,

$$
\exists T_{n} \in R(G) \text { such that } T_{n} \longrightarrow T \text { in norm. }
$$

We assume that $G$ is amenable, so we have a Reiter sequence $f_{m}$ and we apply the averaging to each $u_{n}$ for all $T \in C_{u}^{*}(G)$, get $u_{n} \in R(G)$

$$
u_{n}^{m}(x, y)=\sum_{g \in G} f_{m}\left(x y^{-1}\right)(g) u_{n}(x g, y g)
$$

Thus

$$
\overline{u_{n}}=\text { weak }-\lim _{m \rightarrow \infty} u_{n}^{m}
$$

and also

$$
\overline{T_{n}}=\text { weak }-\lim _{m \longrightarrow \infty} T_{n}^{m} .
$$

Thus

$$
\left\langle T_{n}^{m} \delta x, \delta y\right\rangle \longrightarrow\left\langle\overline{T_{n}} \delta x, \delta y\right\rangle \text { as } n \longrightarrow \infty
$$

and

$$
\left\langle\left(T_{n}^{m}-\overline{T_{n}}\right) \delta x, \delta y\right\rangle \longrightarrow 0 \text { as } n \longrightarrow \infty
$$

Define the semi norm

$$
P_{x y}(T)=\langle T \delta x, \delta y\rangle
$$

Then

$$
P_{x y}\left(T_{n}^{m}-\overline{T_{n}}\right)=\left\langle\left(T_{n}^{m}-\overline{T_{n}}\right) \delta x, \delta y\right\rangle \longrightarrow 0 \text { as } n \longrightarrow \infty
$$

and

$$
P_{x y}\left(T_{n}-T\right)=\left\langle\left(T_{n}-T\right) \delta x, \delta y\right\rangle \longrightarrow 0 \text { as } n \longrightarrow \infty
$$

Since

$$
\left|u_{n}(x g, y g)\right| \leq M \text { for all } \in G
$$

Consider

$$
\begin{aligned}
\left|u_{n}^{m}(x, y)-u_{n}(x g, y g)\right| & =\left|\sum_{g \in G} f_{m}\left(x y^{-1}\right)(g) u_{n}(x g, y g)-u_{n}(x g, y g)\right| \\
& \leq\left\{\sum_{g \in G}\left|f_{m}\left(x y^{-1}\right)(g)\right|+1\right\}\left|u_{n}(x g, y g)\right| \\
& =\left\{\left\|f_{m}\right\|_{\ell^{1}}+1\right\}\left|u_{n}(x g, y g)\right| \\
& \leq 2 M .
\end{aligned}
$$


Thus $u_{n}^{m}(x, y)$ does not convege to $u_{n}(x g, y g)$ as $m \longrightarrow \infty$. Consider

$$
\begin{gathered}
\sum_{g \in G} f_{m}\left(x y^{-1}\right)\left\langle T_{n}^{m} \delta x, \delta y\right\rangle-\sum_{g \in G} f_{m}\left(x y^{-1}\right)\left\langle T_{n} \delta x, \delta y\right\rangle \\
\sum_{g \in G} f_{m}\left(x y^{-1}\right)\left\langle\left(T_{n}^{m}-T_{n}\right) \delta x, \delta y\right\rangle
\end{gathered}
$$

This does not convege to zero as $m \longrightarrow \infty$. Therefore

$$
P_{x y}\left(T_{n}^{m}-T_{n}\right)=\left\langle T_{n}^{m}-T_{n} \delta x, \delta y\right\rangle \text { does not converge } 0 \text { as } n \longrightarrow \infty .
$$

consider

$$
P_{x y}\left(\overline{T_{n}}-T\right) \leq P_{x y}\left(\overline{T_{n}}-T_{n}^{m}\right)++P_{x y}\left(T_{n}^{m}-T_{n}\right)+P_{x y}\left(T_{n}-T\right)
$$

Thus $P_{x y}\left(\overline{T_{n}}-T\right)$ does not converge 0 as $\mathrm{n} \longrightarrow \infty$. Therefore $\overline{T_{n}} \neq T$.

$$
T \notin C_{\rho}^{*}(G),
$$

Since

$$
T \in C_{u}^{*}(G)^{G}
$$

Thus

$$
C_{u}^{*}(G)^{G} \neq C_{\lambda}^{*}(G)
$$

Therefore $G$ hasnot IAP.

\section{References}

[1] C. Anantharaman-Delaroche, Amenable correspondences and approximation properties for von Neumann algebras, Pacific J. Math., 171, No. 2 (1995), 309-341, http://projecteuclid.org/euclid.pjm/1102368918.

[2] J. Brodzki, G. Niblo. P.W. Nowak, N. Wright, Amenable actions, invariant means and bounded cohomology, J. Topol. Anal., 04, No. 321 (2012), 321334, doi: 10.1142/S1793525312500161.

[3] J. Brodzki, G. Niblo, P.W. Nowak, N. Wright, Pairings, duality, amenability and bounded cohomology, J. of the European Mathematical, 14, No. 4 (2012), 1513-1518, doi: 10.4171/JEMS/338.

[4] K.R. Davidson, $C^{*}$-Algebra by Example, Field Institute Mono-graphs, American Mathematical Soc, Providence, RI, 6 (1986). 
[5] K. Kannan, Strong invariant approximation property for Discrete groups, International Journal of Pure and Applied Mathematics, 85, No. 6 (2013), 1075-1086, doi: 10.12732/ijpam.v85i6.11

[6] K. Kannan, The stability properties of strong invariant approximation property, International Journal of Pure and Applied Mathematics, 88, No. 4 (2013), 557-567, doi: 10.12732/ijpam.v88i4.10.

[7] K. Kannan, On the invariant uniform Roe algebras as crossed product, International Science Index, 7 (2013), 1458-1464, http://www.waset.org/publications/1826

[8] K. Kannan, On approximation properties of group $\mathrm{C}^{*}$ - algebras, University of Southampton, School of Mathematics, Doctoral Thesis (2011), No. 2, $116 \mathrm{pp}$.

[9] J. Roe, Lectures on Coarse Geometry, American Mathematical Society, 31 (2003). 